\title{
Application of the sextic oscillator potential together with Mathieu and spheroidal functions for triaxial and X(5) type nuclei
}

\author{
A. A. Raduta ${ }^{1,2, a}$ and P. Buganu ${ }^{1}$ \\ ${ }^{1}$ Horia Hulubei National Institute of Physics and Nuclear Engineering, Magurele, RO-077125, Romania \\ ${ }^{2}$ Academy of Romanian Scientists, 54 Splaiul Independentei, Bucharest 050094, Romania
}

\begin{abstract}
The Bohr-Mottelson Hamiltonian is amended with a potential which depends on both $\beta$ and $\gamma$ deformation variables and which allows us to separate the $\beta$ variable from the other variables. The equation for the $\beta$ variable is quasi-exactly solved for a sextic oscillator with centrifugal barrier potential. Concerning the $\gamma$ equation, its solutions are the angular spheroidal and Mathieu functions for X(5) type and triaxial nuclei, respectively. The models developed in this way are conventionally called the Sextic and Spheroidal Approach (SSA) and the Sextic and Mathieu Approach (SMA). SSA and SMA was successfully applied for several nuclei, details being presented below.
\end{abstract}

\section{Introduction}

A great interest in solving the eigenvalue problem of the Bohr-Mottelson Hamiltonian [1] having a potential which depends on both $\beta$ and $\gamma$ variables appeared when nuclei being close to the critical points of some shape phase transition were very well described by analytically solvable equations. The E(5) [2] solution describes the critical point of the transition between spherical and $\gamma$-unstable shape phases, while the one associated to the transition between spherical and axially symmetric shape phases is called $\mathrm{X}(5)$ [3]. Other two solutions for critical points were proposed short after that, namely $\mathrm{Y}(5)$ [4] and $\mathrm{Z}(5)$ [5], for the axial-triaxial shape phase transition and for the prolate-oblate shape phase transition, respectively.

In the this paper, we present new interesting solutions for the Hamiltonian [1], namely, Sextic and Mathieu Approach (SMA) [6-8] and Sextic and Spheroidal Approach (SSA) [9], respectively. SMA represents a realistic tool for the description of triaxial nuclei having axial deformations close to $\pi / 6$, while SSA works very well for X(5) type and axial nuclei.

\section{New solutions for the generalized Bohr-Mottelson Hamiltonian}

The Bohr-Mottelson Hamiltonian [1],

$$
\hat{H}=-\frac{\hbar^{2}}{2 B}\left[\frac{1}{\beta^{4}} \frac{\partial}{\partial \beta} \beta^{4} \frac{\partial}{\partial \beta}+\frac{1}{\beta^{2}}\left(\frac{1}{\sin 3 \gamma} \frac{\partial}{\partial \gamma} \sin 3 \gamma \frac{\partial}{\partial \gamma}-\sum_{k=1}^{3} \frac{\hat{Q}_{k}^{2}}{4 \sin ^{2}\left(\gamma-\frac{2 \pi}{3} k\right)}\right)\right]+V(\beta, \gamma),
$$

\footnotetext{
a e-mail: raduta@nipne.ro
} 
is amended with a potential which depends on both $\beta$ and $\gamma$ deformation variables and which allows us to separate the $\beta$ variable from the $\gamma$ variable and the three Euler angles $\theta_{1}, \theta_{2}$ and $\theta_{3}$, which are still coupled due to the rotational term. Here, with $Q_{k}$ are denoted the intrinsic angular momentum components. Further, by performing a second order expansion of the rotational term around $\gamma_{0}=0$ and $\gamma_{0}=\pi / 6$ for $\mathrm{X}(5)$ type nuclei and triaxial nuclei respectively, and then averaging the resulting terms with specific Wigner functions, a complete separation is achieved [6, 9]:

$$
\begin{gathered}
{\left[-\frac{1}{\beta^{4}} \frac{d}{\partial \beta} \beta^{4} \frac{d}{d \beta}+\frac{L(L+1)}{\beta^{2}}+v_{1}(\beta)\right] f(\beta)=\varepsilon_{\beta} f(\beta), v_{1}(\beta)=\frac{2 B}{\hbar^{2}} V_{1}(\beta), \varepsilon_{\beta}=\frac{2 B}{\hbar^{2}} E_{\beta}} \\
{\left[-\frac{1}{\sin 3 \gamma} \frac{d}{d \gamma} \sin 3 \gamma \frac{d}{d \gamma}-\tilde{W}(L, K, R)+v_{2}(\gamma)\right] \phi(\gamma)=\tilde{\varepsilon}_{\gamma} \phi(\gamma), v_{2}(\gamma)=\frac{2 B}{\hbar^{2}} V_{2}(\gamma), \tilde{\varepsilon}_{\gamma}=\left\langle\beta^{2}\right\rangle \frac{2 B}{\hbar^{2}} E_{\gamma} .}
\end{gathered}
$$

$\tilde{W}(L, K, R)$ contains the terms coming from the rotational term expansion and $L(L+1), R$ and $K$ are the eigenvalues of the total intrinsic angular momentum $\hat{Q}$ and of its projections on the axis 1 and 3 .

Making the change of function $f(\beta)=\beta^{-2} \varphi(\beta)$ and considering a sextic oscillator potential,

$$
v_{1}^{ \pm}(\beta)=\left(b^{2}-4 a c^{ \pm}\right) \beta^{2}+2 a b \beta^{4}+a^{2} \beta^{6}+u_{0}^{ \pm}, \quad c^{ \pm}=\frac{L}{2}+\frac{5}{4}+M, M=0,1,2, \ldots,
$$

Eq. (2) is exactly solved. The meaning of the term quasi-exactly is given in Ref. [6]. Here, $c$ is a constat which has two different values, one for $L$ even and other for $L$ odd, while $u_{0}^{ \pm}$are constants that are fixed such that the potential for $L$ odd to have the same minimum energy with the potential for $L$ even. The solutions for the $\beta$ variable are

$$
\varphi_{n_{\beta}, L}^{(M)}(\beta)=N_{n_{\beta}, L} P_{n_{\beta}, L}^{(M)}\left(\beta^{2}\right) \beta^{L+1} e^{-\frac{a}{4} \beta^{4}-\frac{b}{2} \beta^{2}}, E_{\beta}=\frac{\hbar^{2}}{2 B}\left[b(2 L+3)+\lambda_{n_{\beta}}^{(M)}(L)+u_{0}^{ \pm}\right], n_{\beta}=0,1, \ldots, M,
$$

where $P_{n_{\beta}, L}^{(M)}\left(\beta^{2}\right)$ are polynomials in $\beta^{2}$ of $n_{\beta}$ order and eigenvectors of the equation:

$$
\left[-\frac{d^{2}}{d \beta^{2}}-\frac{4 s-1}{\beta} \frac{d}{d \beta}+2 b \beta \frac{d}{d \beta}+2 a \beta^{2}\left(\beta \frac{d}{d \beta}-2 M\right)\right] P_{n_{\beta}, L}^{(M)}=\lambda_{n_{\beta}}^{(M)} P_{n_{\beta}, L}^{(M)}, s=\frac{L}{2}+\frac{3}{4} .
$$

Concerning Eq. (3), its potential is chosen such that to exhibit minima in $\gamma=0$ and $\gamma=\pi / 6$, namely, $v_{2}(\gamma)=u_{1} \cos 3 \gamma+u_{2} \cos ^{2} 3 \gamma$. Performing a second order expansion around $\gamma_{0}=0$ in $\sin 3 \gamma$ of $v_{2}(\gamma)$ and then substituting $x=\cos 3 \gamma$ and $S(x) \equiv \phi(\gamma)$, we obtain the spheroidal equation [10]:

$$
\begin{gathered}
{\left[\left(1-x^{2}\right) \frac{d^{2}}{d x^{2}}-2 x \frac{d}{d x}+\lambda_{m_{\gamma}, n_{\gamma}}-c^{2} x^{2}-\frac{m_{\gamma}^{2}}{1-x^{2}}\right] S_{m_{\gamma}, n_{\gamma}}(x)=0, m_{\gamma}=\frac{K}{2}, n_{\gamma}=0,1,2, \ldots} \\
\lambda_{m_{\gamma}, n_{\gamma}}=\frac{1}{9}\left(\tilde{\varepsilon}_{\gamma}-\frac{u_{1}}{2}+\frac{L(L+1)+11 K^{2}+22}{12}\right), c^{2}=\frac{1}{9}\left[\frac{u_{1}}{2}+u_{2}-\frac{2}{27}\left(L(L+1)-K^{2}-2\right)\right] .
\end{gathered}
$$

For triaxial nuclei, expanding $v_{2}(\gamma)$ around $\frac{\pi}{6}$, after some steps, we get the Mathieu equation [8]:

$$
\begin{gathered}
\left(\frac{d^{2}}{d y^{2}}+a-2 q \cos 2 y\right) M(y)=0, \quad y=3 \gamma, \phi(\gamma)=\frac{\left|M_{L, R, n_{\gamma}}(3 \gamma)\right|^{2}}{|\sin 3 \gamma|} \\
q=\frac{1}{36}\left[\frac{10}{9} L(L+1)-\frac{13}{12} R^{2}+u_{1}-\frac{9}{4}\right], a=\frac{1}{9}\left(\tilde{\varepsilon}_{\gamma}+\frac{3}{4} R^{2}+\frac{5}{2}\right)-2 q .
\end{gathered}
$$

The $\gamma$ functions are normalized to unity with the integration measure $|\sin 3 \gamma| d \gamma$ :

$$
\frac{3\left(2 n_{\gamma}+1\right)\left(n_{\gamma}-m_{\gamma}\right) !}{2\left(n_{\gamma}+m_{\gamma}\right) !} \int_{0}^{\frac{\pi}{3}}\left|S_{m_{\gamma}, n_{\gamma}}(\cos 3 \gamma)\right|^{2}|\sin 3 \gamma| d \gamma=1, \frac{6}{\pi} \int_{0}^{\frac{\pi}{3}} \frac{\left|M_{L, R, n_{\gamma}}(3 \gamma)\right|^{2}}{|\sin 3 \gamma|}|\sin 3 \gamma| d \gamma=1 .
$$


The total energy of the system is obtained by adding the eigenvalues of the $\beta$ and $\gamma$ equations.

The reduced E2 transition probabilities are determined using the following transition operator:

$$
T_{2 \mu}^{(E 2)}=t_{1} \beta\left[\cos \gamma D_{\mu 0}^{2}+\frac{\sin \gamma}{\sqrt{2}}\left(D_{\mu 2}^{2}+D_{\mu,-2}^{2}\right)\right]+t_{2} \sqrt{\frac{2}{7}} \beta^{2}\left[-\cos 2 \gamma D_{\mu 0}^{2}+\frac{\sin 2 \gamma}{\sqrt{2}}\left(D_{\mu 2}^{2}+D_{\mu,-2}^{2}\right)\right] .
$$

For triaxial nuclei, in Eq. (12) $\gamma$ is substituted with $\gamma-2 \pi / 3$. The argument is justified by the fact that $\gamma-2 \pi / 3$ defines the axis 1 of the principal inertial ellipsoid. Indeed, the transformation from the laboratory to the intrinsic frame is a rotation defined by the matrix $D_{M R}^{L}$, where $\mathrm{M}$ and $\mathrm{R}$ are eigenvalues of the operator $\hat{Q}_{1}$. The models developed in this way are conventionally called the Sextic and Spheroidal Approach (SSA) [9] and the Sextic and Mathieu Approach (SMA) [6-8], respectively.

\section{Numerical results}

In Refs. [6-8], the SMA was successfully applied for several triaxial nuclei, ${ }^{188,190,192} \mathrm{Os},{ }^{228,230} \mathrm{Th}$, ${ }^{180} \mathrm{Hf}$ and ${ }^{182} \mathrm{~W}$, chosen according to a certain signature of the rigid triaxial rotor. In Ref. [9], a good agreement of the SSA results with experimental data of several X(5) candidate nuclei as $176,178,180,188,190 \mathrm{Os},{ }^{150} \mathrm{Nd},{ }^{170} \mathrm{~W},{ }^{156} \mathrm{Dy}$ and ${ }^{166,168} \mathrm{Hf}$, was obtained. In Ref. [9], the SSA results were compared with those yielded by X(5), ISW [10], D [10] and Coherent State Model (CSM) [11]. From space reasons we present here only one nucleus for each of the models, SMA and SSA. For SMA, the numerical results for ${ }^{192}$ Os are shown in Fig. 1 and Table 1. Both, energy spectrum and reduced probability transitions are very well explained by the SMA and CSM. Also, the staggering behavior [15] of the $\gamma$ band is reproduced by the SMA. Comparing the results for ${ }^{188}$ Os presented in Tables 2,3 , we can see that the best agreement with experimental data, for both energy spectrum and E2 transition probabilities, is obtained with SSA.

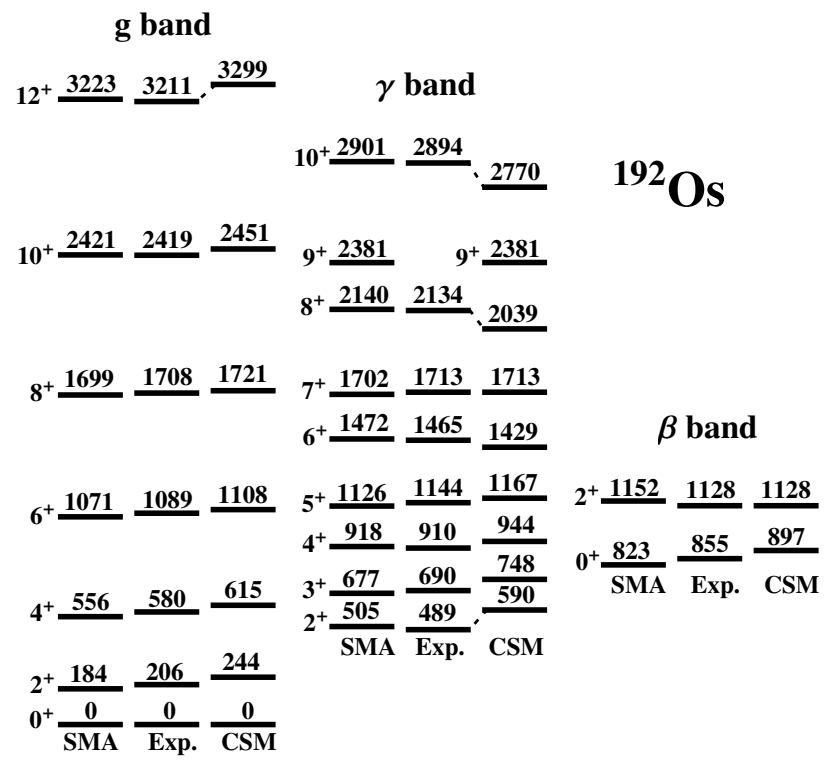

Figure 1: Excitation energies, given in $\mathrm{keV}$, for ground, $\beta$ and $\gamma$ bands for ${ }^{192} \mathrm{Os}$, calculated with SMA and CSM, are compared with the experimental data $[12,13]$.

\begin{tabular}{cccc}
\hline$B\left(E 2 ; J_{i}^{+} \rightarrow J_{f}^{+}\right)$ & Exp. & SMA & CSM \\
\hline $2_{g}^{+} \rightarrow 0_{g}^{+}$ & 0.424 & 0.424 & 0.236 \\
$4_{g}^{+} \rightarrow 2_{g}^{+}$ & 0.497 & 0.632 & 0.449 \\
$6_{g}^{+} \rightarrow 4_{g}^{+}$ & 0.660 & 0.858 & 0.611 \\
$8_{g}^{+} \rightarrow 6_{g}^{+}$ & 0.754 & 1.030 & 0.754 \\
$10_{g}^{+} \rightarrow 8_{g}^{+}$ & 0.688 & 1.175 & 0.887 \\
$4_{\gamma}^{+} \rightarrow 2_{\gamma}^{+}$ & 0.298 & 0.261 & 0.277 \\
$6_{\gamma}^{+} \rightarrow 4_{\gamma}^{+}$ & 0.336 & 0.352 & 0.595 \\
$8_{\gamma}^{+} \rightarrow 6_{\gamma}^{+}$ & 0.314 & 0.549 & 0.814 \\
$2_{\gamma}^{+} \rightarrow 0_{g}^{+}$ & 0.037 & 0.006 & 0.192 \\
$2_{\gamma}^{+} \rightarrow 2_{g}^{+}$ & 0.303 & 0.303 & 0.055 \\
$2_{\gamma}^{+} \rightarrow 4_{g}^{+}$ & 0.024 & 0.000 & 0.000 \\
$4_{\gamma}^{+} \rightarrow 2_{g}^{+}$ & 0.002 & 0.004 & 0.274 \\
$4_{\gamma}^{+} \rightarrow 4_{g}^{+}$ & 0.203 & 0.068 & 0.137 \\
$4_{\gamma}^{+} \rightarrow 6_{g}^{+}$ & 0.018 & 0.000 & 0.000 \\
$6_{\gamma}^{+} \rightarrow 4_{g}^{+}$ & 0.000 & 0.002 & 0.357 \\
$6_{\gamma}^{+} \rightarrow 6_{g}^{+}$ & 0.171 & 0.042 & 0.171 \\
\hline
\end{tabular}

Table 1: E2 transition probabilities, given in $(\mathrm{eb})^{2}$, for ${ }^{192} \mathrm{Os}$, calculated with SMA and CSM, are compared with the experimental data $[12,13]$. 


\begin{tabular}{ccccccc}
\hline${ }^{188} \mathrm{Os}$ & Exp. & $\mathrm{X}(5)$ & $\mathrm{ISW}$ & $\mathrm{D}$ & SSA & $\mathrm{CSM}$ \\
\hline $2_{g}^{+}$ & 155 & 179 & 179 & 151 & 152 & 150 \\
$4_{g}^{+}$ & 478 & 519 & 519 & 479 & 476 & 468 \\
$6_{g}^{+}$ & 940 & 970 & 970 & 945 & 935 & 934 \\
$8_{g}^{+}$ & 1515 & 1516 & 1516 & 1512 & 1501 & 1535 \\
$10_{g}^{+}$ & 2170 & 2149 & 2150 & 2156 & 2154 & 2264 \\
$12_{g}^{+}$ & 2856 & 2867 & 2868 & 2860 & 2877 & 3116 \\
$0_{\beta}^{+}$ & 1086 & 1009 & 1007 & 1120 & 1063 & 1164 \\
$2_{\beta}^{+}$ & 1305 & 1331 & 1328 & 1270 & 1330 & 1305 \\
$4_{\beta}^{+}$ & & 1910 & 1907 & 1599 & 1808 & 1621 \\
$6_{\beta}^{+}$ & & 2636 & 2632 & 2064 & 2421 & 2096 \\
$8_{\beta}^{+}$ & & 3474 & 3470 & 2632 & 3132 & 2717 \\
$10_{\beta}^{+}$ & & 4412 & 4407 & 3276 & 3920 & 3475 \\
$2_{\gamma}^{+}$ & 633 & 631 & 631 & 627 & 641 & 665 \\
$3_{\gamma}^{+}$ & 790 & 786 & 785 & 773 & 791 & 790 \\
$4_{\gamma}^{+}$ & 966 & 972 & 971 & 959 & 969 & 956 \\
$5_{\gamma}^{+}$ & 1181 & 1185 & 1185 & 1180 & 1172 & 1157 \\
$6_{\gamma}^{+}$ & 1425 & 1423 & 1423 & 1432 & 1434 & 1399 \\
$7_{\gamma}^{+}$ & 1686 & 1685 & 1684 & 1709 & 1674 & 1669 \\
$8_{\gamma}^{+}$ & & 1969 & 1969 & 2009 & 2008 & 1983 \\
$9_{\gamma}^{+}$ & & 2275 & 2275 & 2329 & 2273 & 2318 \\
$10_{\gamma}^{+}$ & & 2602 & 2603 & 2666 & 2670 & 2701 \\
\hline r.m.s. $[\mathrm{keV}]$ & & 27 & 27 & 16 & 13 & 36 \\
\hline & & & & & &
\end{tabular}

Table 2: The energy spectrum, given in $\mathrm{keV}$, of the ground, $\beta$ and $\gamma$ bands for ${ }^{188}$ Os yielded by the $\mathrm{X}(5)$, ISW, D, SSA and CSM are compared with the experimental data taken from Ref. [14].

\begin{tabular}{ccccccc}
\hline B(E2)(W.u.) & Exp. & X(5) & ISW & D & SSA & CSM \\
\hline $2_{g}^{+} \rightarrow 0_{g}^{+}$ & $79_{-2}^{+2}$ & 74 & 72 & 79 & 82 & 42 \\
$4_{g}^{+} \rightarrow 2_{g}^{+}$ & $133_{-8}^{+8}$ & 118 & 115 & 121 & 123 & 87 \\
$6_{g}^{+} \rightarrow 4_{g}^{+}$ & $138_{-8}^{+8}$ & 147 & 144 & 147 & 145 & 125 \\
$8_{g}^{+} \rightarrow 6_{g}^{+}$ & $161_{-11}^{+11}$ & 169 & 166 & 174 & 162 & 161 \\
$10_{g}^{+} \rightarrow 8_{g}^{+}$ & $188_{-25}^{+25}$ & 187 & 184 & 203 & 178 & 195 \\
$0_{\beta}^{+} \rightarrow 2_{g}^{+}$ & $0.95_{-0.08}^{+0.08}$ & 47 & 48 & 33 & 21 & 0.95 \\
$0_{\beta}^{+} \rightarrow 2_{\gamma}^{+}$ & $4.3_{-0.5}^{+0.5}$ & 5.2 & 5.2 & 1.9 & 1.5 & 44 \\
$4_{\gamma}^{+} \rightarrow 2_{\gamma}^{+}$ & $47_{-10}^{+10}$ & 47 & 50 & 52 & 56 & 14 \\
$4_{\gamma}^{+} \rightarrow 3_{\gamma}^{+}$ & $320_{-120}^{+120}$ & 112 & 117 & 120 & 132 & 43 \\
$6_{\gamma}^{+} \rightarrow 4_{\gamma}^{+}$ & $70_{-30}^{+30}$ & 107 & 111 & 114 & 118 & 31 \\
$2_{\gamma}^{+} \rightarrow 0_{g}^{+}$ & $5_{-0.6}^{+0.6}$ & 8.4 & 10.9 & 10.8 & 9.9 & 5 \\
$2_{\gamma}^{+} \rightarrow 2_{g}^{+}$ & $16_{-2}^{+2}$ & 13 & 17 & 16 & 14 & 10.4 \\
$2_{\gamma}^{+} \rightarrow 4_{g}^{+}$ & $34_{-5}^{+5}$ & 0.65 & 0.85 & 0.80 & 0.73 & 1.4 \\
$4_{\gamma}^{+} \rightarrow 2_{g}^{+}$ & $1.29_{-0.19}^{+0.19}$ & 5.7 & 7.1 & 6.7 & 6.1 & 1.7 \\
$4_{\gamma}^{+} \rightarrow 4_{g}^{+}$ & $19_{-3}^{+3}$ & 18 & 23 & 20 & 19 & 10.7 \\
$4_{\gamma}^{+} \rightarrow 6_{g}^{+}$ & $16_{-7}^{+7}$ & 2 & 2 & 2 & 2 & 5 \\
$6_{\gamma}^{+} \rightarrow 4_{g}^{+}$ & $0.21_{-0.11}^{+0.11}$ & 5.3 & 6.4 & 5.8 & 5.3 & 0.9 \\
$6_{\gamma}^{+} \rightarrow 6_{g}^{+}$ & $>9.4$ & 21 & 25 & 23 & 20 & 8.3
\end{tabular}

Table 3: The reduced E2 transition probabilities determined with the X(5), ISW, D, SSA and CSM models for ${ }^{188}$ Os are compared with the corresponding experimental data taken from Ref. [14].

\section{Conclusions}

SSA and SMA represent realistic tools for the description of X(5) type and triaxial nuclei. The Mathieu and spheroidal functions are periodic, defined on bounded intervals and normalized to unity with the integration measure $|\sin 3 \gamma| d \gamma$, preserving in this way the hermiticity of the initial $\gamma$ Hamiltonian. CSM works very well also for nuclei being in critical points of the shape phase transitions.

\section{References}

[1] A. Bohr, B.Mottelson, Mat. Fys. Medd. Dan. Vid. Selsk. 26, no.14 (1952); 27 no. 16 (1953).

[2] F. Iachello, Phys. Rev. Lett. 85, 3580 (2000).

[3] F. Iachello, Phys. Rev. Lett. 87, 052502 (2001).

[4] F. Iachello, Phys. Rev. Lett. 91, 132502 (2003).

[5] D. Bonatsos, D. Lenis, D. Petrellis, P. A. Terziev, Phys. Lett B 588, 172 (2004).

[6] A. A. Raduta, P. Buganu, Phys. Rev. C 83, 034313 (2011).

[7] P. Buganu, A. A. Raduta, A. Faessler, J. Phys. G: Nucl. Part. Phys. 39, 025103 (2012).

[8] P. Buganu, A. A. Raduta, Rom. Journ. Phys. 57, 1103-1112 (2012).

[9] A. A. Raduta, P. Buganu, J. Phys. G: Nucl. Part. Phys. 40, 025108 (2013).

[10] A. A. Raduta, A. C. Gheorghe, P. Buganu, A. Faessler, Nucl. Phys. A 819, 46 (2009).

[11] A. A. Raduta, V. Ceausescu, A. Gheorghe, R. M. Dreizler, Phys. Lett. B 99, 444 (1981).

[12] C. Y. Wu et al.,, Nucl. Phys. A 607, 178 (1996).

[13] Coral M. Baglin, Nuclear Data Sheets 84, 717 (1998).

[14] Balraj Singh, Nuclear Data Sheets 95, 387 (2002).

[15] A. A. Raduta, C. Sabac, Ann. Phys. (NY) 148, 1 (1983). 